\title{
Missing baryonic resonances in the Hagedorn spectrum ${ }^{\star}$
}

\author{
Pok Man Lo ${ }^{1,2, a}$, Michał Marczenko ${ }^{1}$, Krzysztof Redlich ${ }^{1,2,3}$, and Chihiro Sasaki ${ }^{1}$ \\ 1 Institute for Theoretical Physics, University of Wrocław, 50-204 Wrocław, Poland \\ 2 Extreme Matter Institute EMMI, GSI, Planckstrasse 1, D-64291 Darmstadt, Germany \\ 3 Department of Physics, Duke University, Durham, NC 27708, USA
}

Received: 23 December 2015 / Revised: 18 April 2016

Published online: 19 August 2016

(c) The Author(s) 2016. This article is published with open access at Springerlink.com

Communicated by D. Blaschke

\begin{abstract}
The hadronic medium of $\mathrm{QCD}$ is modeled as a gas of point-like hadrons, with its composition determined by the Hagedorn mass spectrum. The spectrum consists of a discrete and a continuous part. The former is determined by the experimentally confirmed resonances tabulated by the Particle Data Group (PDG), while the latter can be extracted from the existing lattice data. This formulation of the hadron resonance gas (HRG) provides a transparent framework to relate the fluctuation of conserved charges as calculated in the lattice QCD approach to the particle content of the medium. A comparison of the two approaches shows that the equation of state is well described by the standard HRG model, which includes only a discrete spectrum of known hadrons. The corresponding description in the strange sector, however, shows clear discrepancies, thus a continuous spectrum is added to incorporate the effect of missing resonances. We propose a method to extract the strange-baryon spectrum from the lattice data. The result is consistent with the trend set by the unconfirmed strange baryons resonances listed by the PDG, suggesting that most of the missing interaction strength for the strange baryons reside in the $|S|=1$ sector. This scenario is also supported by recent lattice calculations, and might be important in the energy region covered by the NICA accelerator in Dubna, where in the heavy-ion collisions, baryons are the dominating degrees of freedom in the final state.
\end{abstract}

\section{Introduction}

One of the consequences of confinement is that physical observables admit a representation in terms of the hadronic states. This means that at low temperature the theory can be written in terms of the ground-state hadrons and their resonances. Such an approach to QCD is adopted by the hadron resonance gas (HRG) model [1-8]. The model assumes that resonance formation dominates the thermodynamics of the confined phase, and, to a first approximation, treats the resonances as point-like particles.

The equation of state for strongly interacting matter at low temperature is well described by the standard HRG model, which includes the list of known hadrons compiled by the Particle Data Group (PDG). This has been verified by recent lattice QCD (LQCD) calculations [9-12]. However, the study of LQCD also reveals the limitation of the HRG approach in describing the fluctuation of conserved charges [11]. This is particularly evident in the strange

\footnotetext{
* Contribution to the Topical Issue "Exploring strongly interacting matter at high densities - NICA White Paper" edited by David Blaschke et al.

a e-mail: pmlo@gsi.de
}

sector, where the second-order correlation with the netbaryon number $\left(\chi_{\mathrm{BS}}\right)$ or the strangeness fluctuation $\left(\chi_{\mathrm{SS}}\right)$ are larger in LQCD than those in the HRG model [10,11]. Such deviations are attributed to the missing resonances in the PDG database [11].

Various extensions of the HRG model have been proposed. They account for a possible repulsive interaction among the constituents and/or for the heavy resonances via a continuously growing mass spectrum $[5,6,8,13]$. The latter was first introduced by Hagedorn [14] within the statistical bootstrap model [15-17], and was then studied in dual string and bag models [18-20].

In this paper, we describe a method for extracting a continuous mass spectrum from LQCD. The spectrum, by definition, explains the lattice data in the particular interaction channel. When applied to the strange-baryon sector, the spectrum is consistent with the trend set by the unconfirmed resonances, suggesting that most of the missing strange baryons reside in the $|S|=1$ sector. We also discuss to what extent this scenario is supported by the recent lattice result.

There are theoretical indications that baryons are the dominating degrees of freedom in the heavy-ion collisions 
$[21,22]$, at the collision energies covered by the proposed NICA accelerator in Dubna. Consequently, the missing baryons in the strange sector could be directly identified from the experimental measurements of strangeness fluctuations and the relevant correlations. Such study could also be helpful in establishing the thermal parameters at chemical freezeout in the low energy region.

\section{Hadron resonance gas and LQCD}

In its simplest form, the HRG model treats the medium constituents as point-like and independent. The chemical composition of the medium is specified by a mass spectrum $\rho(m)$. From this all the thermodynamic quantities may be derived.

It is common to construct the spectrum from a list of all hadrons compiled by the PDG [23]. This includes all the experimentally confirmed mesons and baryons of masses up to $m_{M} \simeq 2.4 \mathrm{GeV}$ and $m_{B} \simeq 2.6 \mathrm{GeV}$, respectively. The list keeps growing as more and more resonances are discovered by the experiment, although the precise determination of their properties remains challenging due to their complicated decay properties.

Within this approximation, the spectrum takes the following discrete form:

$$
\rho^{\mathrm{HRG}}(m)=\sum_{i} d_{i} \delta\left(m-m_{i}\right),
$$

where $d_{i}=\left(2 J_{i}+1\right)$ is the spin degeneracy factor of a particle $i$ with mass $m_{i}$ and the sum is taken over all stable particles and resonances. It is also convenient to define a cumulant function $N^{\mathrm{HRG}}(m)$, which counts the number of degrees of freedom with masses below $m$. The expression reads

$$
N^{\mathrm{HRG}}(m)=\sum_{i} d_{i} \theta\left(m-m_{i}\right)
$$

such that

$$
\rho^{\mathrm{HRG}}=\frac{\partial N^{\mathrm{HRG}}}{\partial m} .
$$

Although the interaction among the medium constituents is neglected in the present approach, the medium itself is far from structureless. The spectrum $\rho(m)$ contains the information about the distributions of mass and quantum number of the medium. Thus, it is possible to study the thermodynamics and even the fluctuations of conserved charges, regardless of the non-interacting nature of the constituents. For example, the pressure $\hat{P}=P / T^{4}$ of an uncorrelated gas of particles (and antiparticles) with a mass spectrum $\rho(m)$ is given by

$$
\begin{aligned}
\hat{P}(T, V, \boldsymbol{\mu})= & \pm \int \mathrm{d} m \rho(m) \int \frac{\mathrm{d} \hat{p}}{2 \pi^{2}} \hat{p}^{2} \\
& \times\left[\ln \left(1 \pm \lambda e^{-\hat{\epsilon}}\right)+\ln \left(1 \pm \lambda^{-1} e^{-\hat{\epsilon}}\right)\right],
\end{aligned}
$$

where $\hat{p}=p / T, \hat{m}=m / T, \hat{\epsilon}=\sqrt{\hat{p}^{2}+\hat{m}^{2}}$, and the $( \pm)$ sign refers to fermions and bosons respectively. For a particle of mass $m$, carrying baryon number $B$, strangeness
$S$ and electric charge $Q$, the fugacity $\lambda$ reads

$$
\lambda(T, \boldsymbol{\mu})=\exp \left(B \hat{\mu}_{B}+S \hat{\mu}_{S}+Q \hat{\mu}_{Q}\right),
$$

where $\hat{\mu}=\mu / T$. Note that for scalar particles with vacuum quantum number, the antiparticle term should be dropped to avoid double counting.

The generalized susceptibilities measure the fluctuations of conserved charges. The second-order fluctuation is defined as

$$
\hat{\chi}_{x y}=\frac{\partial^{2} \hat{P}}{\partial \hat{\mu}_{x} \partial \hat{\mu}_{y}},
$$

where $(x, y)$ are conserved charges, which are restricted to the baryon number $B$ and strangeness $S$ in this study.

In the non-interacting limit, taking the derivative with respect to a given chemical potential naturally selects the spectrum possessing the appropriate quantum number. Thus, $\hat{\chi}_{x y}$ can be used to identify the contribution of different particle species to the thermodynamics $[24,25]$. The explicit expressions of these susceptibilities (at vanishing chemical potentials) within the Boltzmann approximation read

$$
\begin{aligned}
\hat{\chi}_{\mathrm{BB}}^{H}= & \int_{0}^{\infty} \frac{\mathrm{d} m}{\pi^{2}} \rho_{B}(m) \hat{m}^{2} K_{2}(\hat{m}), \\
\hat{\chi}_{\mathrm{SS}}^{H}= & \int_{0}^{\infty} \frac{\mathrm{d} m}{\pi^{2}}\left[\rho_{M}^{S=-1}(m)+\sum_{k=1}^{3} k^{2} \rho_{B}^{S=-k}(m)\right] \\
& \times \hat{m}^{2} K_{2}(\hat{m}), \\
\hat{\chi}_{\mathrm{BS}}^{H}= & -\int_{0}^{\infty} \frac{\mathrm{d} m}{\pi^{2}}\left[\sum_{k=1}^{3} k \rho_{B}^{S=-k}(m)\right] \hat{m}^{2} K_{2}(\hat{m}) .
\end{aligned}
$$

The HotQCD Collaboration [10] and the BudapestWuppertal Collaboration $[9,12]$ have obtained continuumextrapolated results on the thermodynamic pressure (4) and various second-order susceptibilities (7). To test the efficacy of the HRG approach, we compare the lattice results with the predictions of the HRG model formulated with the discrete mass spectrum (1). The results are shown in figs. 1 and 2 .

From fig. 1, it is clear that pressure and net-baryon number fluctuation are well described by the HRG in the hadronic phase, whereas the observables $\hat{\chi}_{\mathrm{BS}}$ in fig. 2(a) and $\hat{\chi}_{\text {SS }}$ in fig. 2 (b) are underestimated in the low temperature phase. Following an analysis of the relations between different susceptibilities of conserved charges, it was argued [10] that deviations seen in fig. 2(a) can be attributed to the missing resonances in the strange baryon sector. In view of fig. 2(b), a similar conclusion can be drawn for the strange mesons.

\section{Spectrum of strange baryons from LQCD}

As described in the previous section, the existing strangebaryon data in the PDG cannot provide sufficient interaction strength in the corresponding channel. Within the 


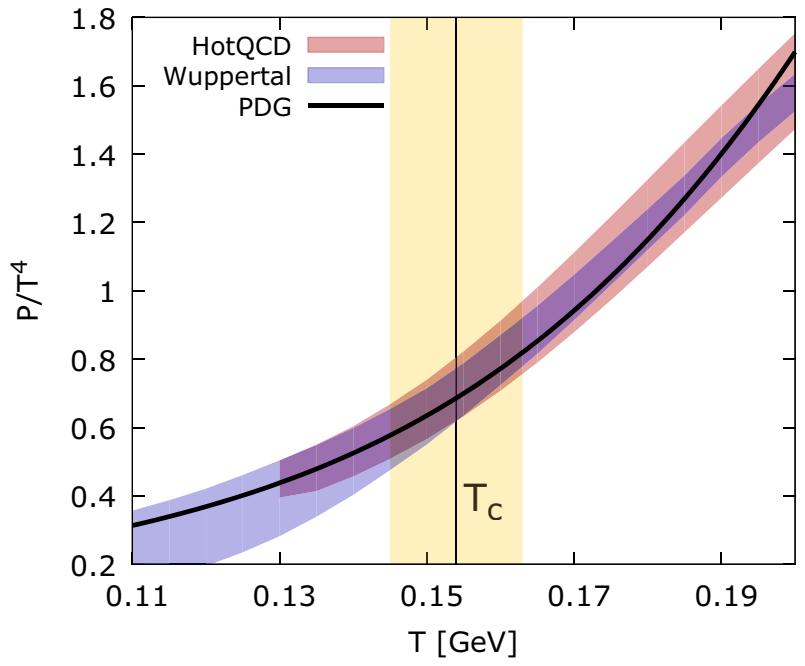

(a)

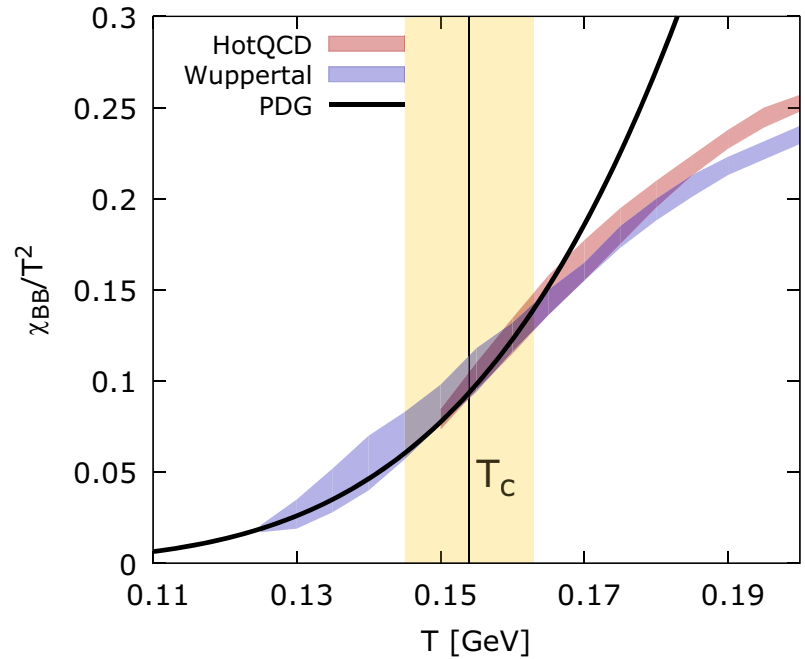

(b)

Fig. 1. (Color online) Lattice QCD results of HotQCD [10,26] and Budapest-Wuppertal Collaborations [9, 12] for different observables in dimensionless units: (a) the thermodynamic pressure; (b) the net-baryon number fluctuations $\hat{\chi}_{\mathrm{BB}}$. Also shown are the HRG results for the discrete PDG mass spectrum (dashed line). See ref. [27].

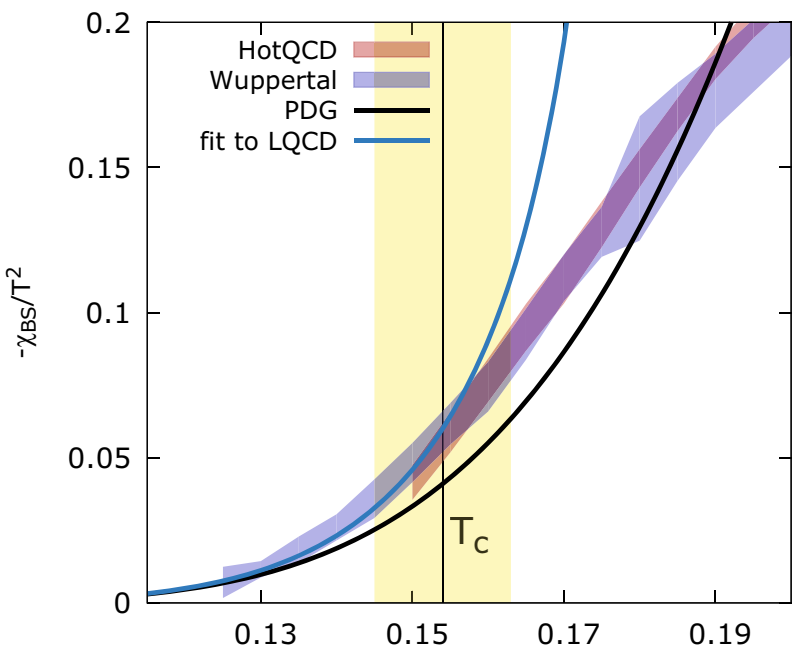

(a)

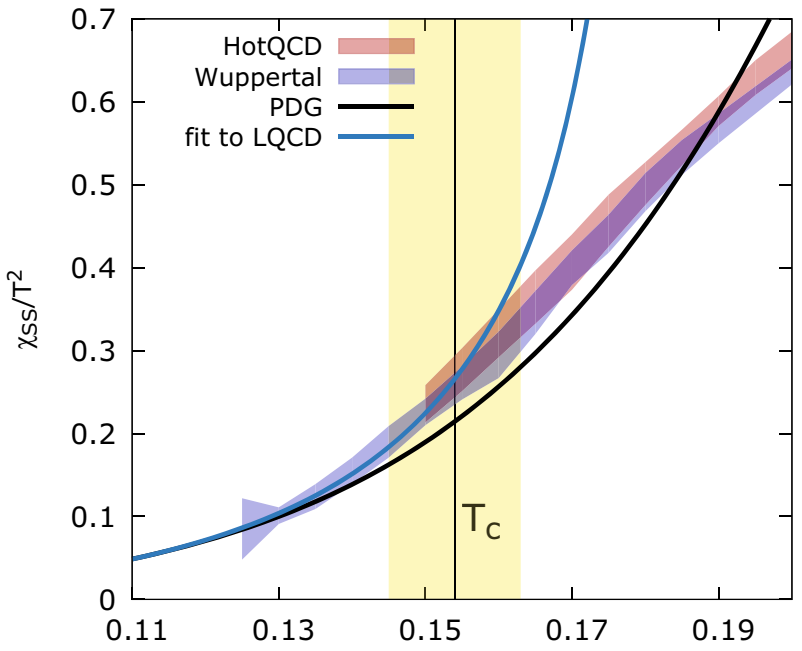

(b)

Fig. 2. (Color online) As in fig. 1, but for baryon-strangeness correlations $\hat{\chi}_{\mathrm{BS}}$ (a) and for strangeness fluctuations $\hat{\chi}_{\mathrm{SS}}(\mathrm{b})$. Also shown are the corresponding results obtained with a model spectrum fitted to lattice data. (See text).

HRG approach, this translates directly to the existence of some missing strange baryons in the mass spectrum.

There are two obvious sources for these missing resonances: heavy resonances beyond the current experimental reach and the strange hadrons excluded by the PDG due to difficulties in their identifications. In both cases, the effect of the missing resonances can be incorporated by augmenting the spectrum $\rho$ with a continuous part. Following the analysis of ref. [27], we employ the following parametrization:

$$
\rho^{H}(m)=\frac{a_{0}}{\left(m^{2}+m_{0}^{2}\right)^{5 / 4}} e^{m / T_{H}}
$$

and its corresponding cumulant

$$
N^{H}(m)=\int_{0}^{m} \mathrm{~d} m^{\prime} \rho^{H}\left(m^{\prime}\right)
$$

where $T_{H} \approx 180 \mathrm{MeV}$ is the Hagedorn limiting temperature, whereas $a_{0}$ and $m_{0}$ are additional model parameters.

Such a continuous functional form works best for a densely populated spectrum. Hence, we adopt Hagedorn's idea to treat the contributions of ground-state particles separately from the exponential mass spectrum. The resulting spectrum reads

$$
\rho(m) \longrightarrow \sum_{i} d_{i} \delta\left(m-m_{i}\right)+\rho^{H}(m) \theta\left(m-m_{x}\right),
$$

and the corresponding cumulant becomes

$$
N(m)=\sum_{i} d_{i} \theta\left(m-m_{i}\right)+\theta\left(m-m_{x}\right) \int_{m_{x}}^{m} \mathrm{~d} m \rho^{H}(m),
$$


Table 1. Left: model parameters of the mass spectra in eq. (10) for strange baryons $(S=-1)$ and strange mesons $(S=-1)$. The parameters are obtained from a least-square fit to the LQCD data on $\hat{\chi}_{\mathrm{BS}}$ and $\hat{\chi}_{\mathrm{SS}}$ through eq. (7), assuming that the missing strangeness contribution comes solely from $|S|=1$ sector [27]. Also shown are the errors of $m_{0}$ and $a_{0}$ arising from the least-square fit. The Hagedorn temperature has been set to $T_{H}=180 \mathrm{MeV}$. Right: the constraint on the continuous mass spectrum in each sector, given in eq. (11).

\begin{tabular}{|c||c|c||c|c|}
\hline \multicolumn{1}{|c||}{} & \multicolumn{2}{c||}{ Fit to LQCD } & \multicolumn{2}{c|}{ Constraint } \\
\hline & $m_{0}[\mathrm{GeV}]$ & $a_{0}\left[\mathrm{GeV}^{3 / 2}\right]$ & $m_{x}[\mathrm{GeV}]$ & $N^{\mathrm{HRG}}\left(m_{x}\right)$ \\
\hline \hline$\rho_{B}^{S=-1}$ & $0.193(96)$ & $0.067(27)$ & 1.3828 & 20 \\
\hline$\rho_{M}^{S=-1}$ & $0.378(32)$ & $0.099(24)$ & 0.89166 & 5 \\
\hline
\end{tabular}

where $\rho^{H}(m)$ is given by eq. (8). The index $i$ counts the hadronic ground states, i.e., states with masses less than mass $m_{x}$ of the first resonance in the corresponding channel.

In general, the parameters of $\rho(m)$ in eq. (10) can be calculated within a model, e.g., in the statistical bootstrap model $[15,17]$. In the following, we discuss a method to extract the mass spectrum from the lattice data.

The basic idea of the approach is quite straightforward. Instead of computing various thermal observables from a model spectrum, we make use of the corresponding lattice results to constrain the spectrum. In practice, one needs to beware of the extra consistency constraints coming from other sectors [27].

We now apply the method to the strange-baryon sector. From eq. (7), we see that the $\hat{\chi}_{\text {BS }}$ data relate only to a linear combination of the spectra, namely $\rho_{B}^{S}=$ $\rho_{B}^{S=-1}+2 \rho_{B}^{S=-2}+3 \rho_{B}^{S=-3}$. Thus, it does not allow for a unique determination of the contribution from an individual sector. This problem may be resolved with additional lattice data on higher-order strangeness fluctuation, e.g. $\hat{\chi}_{\text {BBSS }}$ and the kurtosis, which involves a different linear combination of the spectra.

In ref. [27], we have analyzed the data on $\hat{\chi}_{\mathrm{BS}}$ under the assumption that the additional strange baryons come solely from the $|S|=1$ sector $^{1}$. This allows for the extraction of the model parameters (8) for the strangebaryon spectrum $\rho_{B}^{S=-1}$ by a fit to the lattice data on $\hat{\chi}_{\mathrm{BS}}$ via eq. (7). The parameters for the strange-meson spectrum $\rho_{M}^{S=-1}$ can be analogously obtained from the $\hat{\chi}_{\text {sS }}$ data after subtracting the corresponding contribution from strange baryons. The resultant parameters are given in table 1. For details, see ref. [27].

In fig. 3 we show the resultant cumulants of such lattice-induced $\rho(m)$, together with the experimental spectra including the unconfirmed states from the PDG. The extracted mass spectrum follows the trend of the unconfirmed states of the PDG, supporting the claim that most of the missing strange baryons lie in the $|S|=1$ sector.

1 The remaining ones are treated with a spectrum fitted to the PDG. See ref. [27].
Recent lattice results lend further support to this claim. A decomposition of the strange-baryon pressure into different strange sectors confirms that most of the missing interaction strength come from the $|S|=1$ sector [28]. The corresponding results in the current model are summarized in fig. 3 .

By comparing the magnitude of the partial pressures (which are equivalent to the normalized strangeness fluctuations within HRG), it is clear that the $|S|=1$ sector dominates the interaction strength. In addition, it has been shown that this sector displays a large deviation between the lattice and the standard HRG results [11]. Thus, the assertion that most of the missing strange baryons lie in the $|S|=1$ sector appears to be justified.

Nevertheless, additional baryon resonances are also expected in the $|S|=2$ and $|S|=3$ sectors. This is apparent from fig. 3 and is also supported by an analysis of the lattice data with quark model hadron resonance gas (QM-HRG) $[11,29]$. These multi-strange states can be important in other fluctuation quantities, and may be experimentally probed in the heavy-ion programme at the planned NICA accelerator in Dubna.

\section{Conclusions}

The hadron resonance gas (HRG) model provides a transparent framework to interpret the lattice QCD (LQCD) data in terms of the particle content of the QCD medium.

Despite the agreement on the equation of state between LQCD and the standard HRG, the study of fluctuation of conserved charges reveals the need for some extra resonances in the strange-baryon sector.

We have proposed a method to extract strange-baryon spectrum from the lattice data. This spectrum, built on the assumption that all the missing strange baryons come from the $|S|=1$ sector, explains the lattice data on $\hat{\chi}_{\mathrm{BS}}$, and is consistent with the trend set by the unconfirmed resonances listed by the Particle Data Group (PDG). Furthermore, a recent lattice analysis of the baryon fluctuation lends further support to the assertion that most of the missing strange baryons lie in the $|S|=1$ sector.

The obvious extension of this work is to include the effect of interaction among the hadrons. A consistent framework to incorporate both the attractive and repulsive interaction channels is provided by the $S$-matrix formalism [30]. Using the empirical scattering phase shifts, the effects of resonance widths and mutual repulsive forces among the hadrons can be systematically included. The study of the thermodynamics of the hadronic phase within this approach has been presented by Venugopalan and Prakash [31].

The additional interaction may influence the conclusion concerning the missing strange resonances. For example, additional strength for $\hat{\chi}_{\mathrm{BS}}$ and $\hat{\chi}_{\mathrm{SS}}$ may come from the explicit interaction among the known strange hadrons in the medium $[32,33]$. Also, the non-strange sector may contribute to these observables via hadronic interactions. These effects should be included in the model. We leave this as a matter of future investigation. 

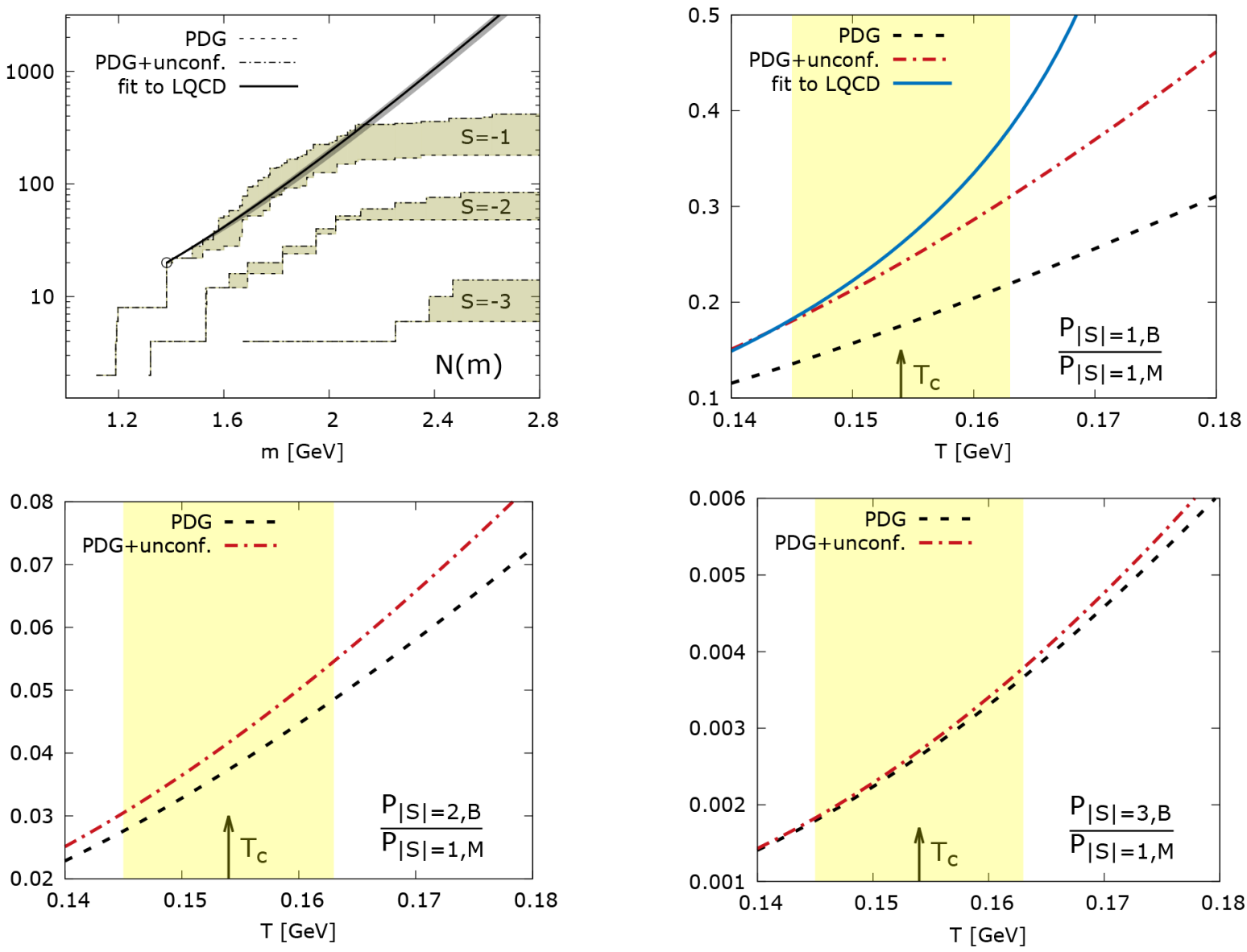

Fig. 3. (Color online) Top left: cumulants of the discrete PDG mass spectrum (black dashed line) for strange baryons. Also shown are the cumulants containing in addition the unconfirmed states (dot-dashed line). Continuous lines are obtained by matching the LQCD results to the continuous mass spectra through eq. (7), assuming that the missing strange baryons come solely from the $|S|=1$ sector (see ref. [27]). Top right and bottom: partial pressures of the strange baryons (normalized to partial pressure of the strange mesons) from different strange sectors, obtained in the current model.

Experimentally, the current study is relevant in describing the medium created in the heavy-ion collision at energies covered by the proposed NICA accelerator in Dubna. In this case, the baryons are expected to be the dominating degrees of freedom in the final state. Thus, the missing strange baryons or other in-medium effects due to hadronic interactions could be directly identified from the experimental measurements of strangeness fluctuations and the relevant correlations.

We acknowledge fruitful discussions with Bengt Friman, Frithjof Karsch and Swagato Mukherjee. PML acknowledges the support of Extreme Matter Institute EMMI. KR also acknowledges fruitful discussion with A. Andronic, S. Bass, P. BraunMunzinger, M. Nahrgang, J. Rafelski, H. Satz and J. Stachel and partial support of the U.S. Department of Energy under Grant No. DE-FG02-05ER41367. This work was partly supported by the Polish National Science Center (NCN), under Maestro Grant DEC-2013/10/A/ST2/00106.
Open Access This is an open access article distributed under the terms of the Creative Commons Attribution License (http://creativecommons.org/licenses/by/4.0), which permits unrestricted use, distribution, and reproduction in any medium, provided the original work is properly cited.

\section{References}

1. P. Braun-Munzinger, K. Redlich, J. Stachel, in QuarkGluon Plasma 3, edited by R.C. Hwa et al. (World Scientific, Singapore, 2004) pp. 491-599.

2. F. Karsch, K. Redlich, A. Tawfik, Eur. Phys. J. C 29, 549 (2003).

3. F. Karsch, K. Redlich, A. Tawfik, Phys. Lett. B 571, 67 (2003).

4. F. Karsch, Acta Phys. Pol. Suppl. 7, 117 (2014).

5. A. Andronic, P. Braun-Munzinger, J. Stachel, M. Winn, Phys. Lett. B 718, 80 (2012).

6. M. Albright, J. Kapusta, C. Young, Phys. Rev. C 90, 024915 (2014). 
7. M. Albright, J. Kapusta, C. Young, Phys. Rev. C 92, 044904 (2015).

8. V. Vovchenko, D.V. Anchishkin, M.I. Gorenstein, Phys. Rev. C 91, 024905 (2015).

9. S. Borsanyi, Z. Fodor, S.D. Katz, S. Krieg, C. Ratti, K. Szabo, JHEP 01, 138 (2012).

10. HotQCD Collaboration (A. Bazavov et al.), Phys. Rev. D 86, 034509 (2012)

11. A. Bazavov, H.-T. Ding, P. Hegde, O. Kaczmarek, F. Karsch, E. Laermann, Y. Maezawa, S. Mukherjee et al., Phys. Rev. Lett. 113, 072001 (2014).

12. S. Borsanyi, Z. Fodor, C. Hoelbling, S.D. Katz, S. Krieg, K.K. Szabo, Phys. Lett. B 730, 99 (2014).

13. A. Majumder, B. Muller, Phys. Rev. Lett. 105, 252002 (2010).

14. R. Hagedorn, Nuovo Cimento Suppl. 3, 147 (1965)

15. R. Hagedorn, CERN yellow report 71-12 (1971).

16. J. Letessier, J. Rafelski, Hadrons and Quark Gluon Plasma, Cambridge Monogr. Part. Phys. Nucl. Phys. Cosmol., Vol. 18 (Cambridge University Press, Cambridge, 2005).

17. S.C. Frautschi, Phys. Rev. D 3, 2821 (1971)

18. K. Huang, S. Weinberg, Phys. Rev. Lett. 25, 895 (1970).

19. J.R. Cudell, K.R. Dienes, Phys. Rev. Lett. 69, 1324 (1992).

20. K. Johnson, C.B. Thorn, Phys. Rev. D 13, 1934 (1976).
21. P. Braun-Munzinger, J. Cleymans, H. Oeschler, K. Redlich, Nucl. Phys. A 697, 902 (2002).

22. J. Randrup, J. Cleymans, Phys. Rev. C 74, 047901 (2006).

23. Particle Data Group Collaboration (K.A. Olive et al.), Chin. Phys. C 38, 090001 (2014).

24. S. Ejiri, C.R. Allton, M. Doring, S.J. Hands, O. Kaczmarek, F. Karsch, E. Laermann, K. Redlich, Nucl. Phys. A $\mathbf{7 7 4}, 837$ (2006).

25. S. Ejiri, F. Karsch, K. Redlich, Phys. Lett. B 633, 275 (2006).

26. HotQCD Collaboration (A. Bazavov et al.), Phys. Rev. D 90, 094503 (2014).

27. P.M. Lo, M. Marczenko, K. Redlich, C. Sasaki, Phys. Rev. C 92, 055206 (2015).

28. A. Bazavov et al., Phys. Rev. Lett. 111, 082301 (2013).

29. S. Mukherjee et al., private communication.

30. R. Dashen, S.K. Ma, H.J. Bernstein, Phys. Rev. 187, 345 (1969).

31. R. Venugopalan, M. Prakash, Nucl. Phys. A 546, 718 (1992).

32. B. Friman, P.M. Lo, M. Marczenko, K. Redlich, C. Sasaki, Phys. Rev. D 92, 074003 (2015).

33. W. Broniowski, F. Giacosa, V. Begun, Phys. Rev. C 92, 034905 (2015). 